\title{
Adaptación y Análisis Psicométrico de la Escala Gilliam para evaluar Trastorno de Asperger
}

\author{
Adaptation and psychometric analysis of the Gilliam Asperger' Disorder Scale
}

\author{
Carmen Gloria Fernández Baeza ${ }^{1}$ \\ Universidad Miguel de Cervantes \\ (Rec: abril 2013 - Acep: septiembre 2013)
}

\begin{abstract}
Resumen
La Escala Gilliam para evaluar Transtorno Asperger (Gilliam Asperger's Disorder Scale, GADS) es una evaluación de calificación conductual, cuyo propósito es ayudar a que los profesionales diagnostiquen el Síndrome de Asperger (SA). Esta escala se utiliza para evaluar sujetos entre los 3 y 22 años y puede ser completada por cualquier persona que tenga contacto regular con un sujeto con SA. Los únicos estudios psicométricos que se conocen con resultados favorables son los de la muestra normativa. Es por esto que esta investigación intenta responder si la GADS conserva las propiedades psicométricas de la muestra normativa al aplicarla en la población chilena con SA. Se contó con una muestra no probabilística intencional compuesta por 36 sujetos en la fase piloto y 111 sujetos en la fase final. Las subescalas obtuvieron un alpha de Cronbach entre 0,73 y 0,87 , mientras que la escala global obtuvo 0,92 . Se realizó un análisis factorial exploratorio, del cual se obtuvo una solución factorial de 8 factores en la fase final. Los resultados permiten concluir que la GADS cuenta con propiedades psicométricas adecuadas, lo que significa que es un instrumento confiable y válido para ser utilizado en la población chilena.
\end{abstract}

Palabras clave: Asperger, GADS, adaptación, análisis psicométrico

\begin{abstract}
The Gilliam Asperger's Disorder Scale (GADS) is a norm-referenced assessment designed to help professionals in Asperger Syndrome (AS) diagnosis. This scale is used to evaluate subjects between 3 and 22 years old and it can be completed by anyone who has regular contact with a subject with AS. The only known psychometric studies with positive results are those of the normative sample. Thus this research attempts to explain whether GADS retains the psychometric properties of the normative sample when applied to the Chilean population with AS. To this purpose it was selected a probabilistic intentional sample compose of 36 subjects in the pilot phase and 111 subjects in the final phase. The subscales obtained a Cronbach's alpha between 0,73 and 0,87, while the global scored 0,92 . It was carried out an exploratory factor analysis, thought which it was obtained a factor solution of 8 in the final phase. The results obtained allow to conclude that GADS has adequate psychometric properties, which means GADS is a valid and reliable instrument to be applied to Chilean population.
\end{abstract}

Key words: Asperger, GADS, adaptation, psychometric analysis

\footnotetext{
Correspondencia a: Carmen Gloria Fernández. E-mail: cgloriaf@ hotmail.com. Teléfonos: 26222610 / 6-6673414
} 


\section{Introducción}

El Síndrome de Asperger (SA) (o Trastorno de Asperger) es el término utilizado para describir la parte más leve y con mayor grado de funcionamiento de los Trastornos Generalizados del Desarrollo (TGD) (Caballero, 2008; Pacheco, Sandoval \& Torrealba, 2008). Se cree que el Síndrome de Asperger es un trastorno del desarrollo de base neurobiológica, de causa desconocida en la mayoría de los casos, donde existen anormalidades en tres aspectos del desarrollo: habilidades sociales, la utilización del lenguaje con fines comunicativos y algunas características de comportamiento relacionados con rasgos repetitivos o perseverantes, además de una intensa pero limitada gama de intereses (Caballero, 2008; Ghaziuddin, 2008; McPartland \&Volkmar, 2009; Rodríguez \& Ridao, 2011). Estos tres tipos de disfunciones, pueden variar en el grado y puede ir desde moderado a severo, estos grados son los que definen clínicamente todos los TGD, desde el Síndrome de Asperger, hasta el autismo clásico de Kanner. La idea de que existe un espectro continuo y unidimensional de los TGD, ayuda a entender las semejanzas clínicas que se presentan a lo largo del espectro (Caballero, 2008).

Una de las principales características de las personas con SA es la ausencia de comprensión social, no entienden los códigos sociales, y pese a que son capaces de aprenderlos si se los enseñan, los obedecen en forma rígida (Attwood, 2009). Esto sucede porque su modo de pensar y actuar resultan rígidos e inflexibles (Alonso, Belinchón, Blanco, Cáceres, de Diego, Frías, Frontera, Giménez, Martínez, Murillo, Nieto \& Saldaña, 2009), y aunque tienen una inteligencia normal e incluso superior, no pueden aplicarla en estas situaciones (Martínez, 2009), ya que su pensamiento es lógico, concreto e hiperrealista (Ramírez, 2010).

Desde un punto de vista epidemiológico, no hay estudios que reflejen unas cifras exactas, ni acuerdo entre los autores de diversas investigaciones, pero en promedio se considera que el SA tiene una prevalencia de 26 a 36 casos por cada 10.000 personas, más frecuente en el sexo masculino que en el femenino (Fernández-Jaén, Fernández-Mayoralas, Calleja-Pérez \& Muñoz, 2007; Marker, Weeks \& Kraegel, 2007; Pacheco, Sandoval \& Torrealba, 2008). En nuestro país, las cifras son desconocidas y no existen investigaciones al respecto (Mella, Araneda, Ormazábal, Salinas \& Sepúlveda, 2011). Como existe un gran desconocimiento de este trastorno, y dado que es una entidad relativamente reciente, muchos casos reciben un diagnóstico equivocado (Fernández, 2011; Ramírez, 2010), y pese a que el SA muestra una incidencia hasta cinco veces más que el autismo, casi la mitad de los casos llegan a la edad adulta sin ser diagnosticados (Fernández-Jaén et al., 2007).

Los TGD y en especial el SA, no son detectables mediante ningún marcador biológico que permita identificarlos y sólo pueden ser diagnosticados a través del estudio de pautas de comportamiento, por lo que la observación minuciosa y la historia que ofrecen los padres son elementos básicos para conocer qué es lo que presenta el niño (Cererols, 2010). De esta manera, el SA se diagnostica en base a unas determinadas pautas conductuales observables, ya sea de manera directa o indirecta, a través de la información recolectada por parte de las personas más cercanas al sujeto (Fernández, 2010). Es necesario recoger datos sobre el desarrollo temprano, los aspectos médicos, educativos, familiares, primeros problemas que se hayan observado, el nivel de expresión verbal, el uso del lenguaje, las áreas de interés anómalas, interacción social, relación con los integrantes de la familia, amistades, etc. (Villanueva, 2011). En este sentido, los cuestionarios tienen un valor clínico importante, ya que hacen posible recabar información de una manera simple y rápida (MartínBorreguero, 2008).

Hay muchos factores involucrados para que la detección del SA no sea frecuente, como la propia definición de los trastornos, la complejidad y heterogeneidad de los síntomas en niños pequeños portadores del síndrome (Cortez \& Contreras, 2007) y las similitudes que tiene con otros trastornos, entre ellos el autismo de alto funcionamiento y el trastorno generalizado del desarrollo no específico (Foley \& Assouline, 2010). De igual forma, influyen la falta de entrenamiento profesional adecuado para la detección temprana y la falta de instrumentos específicos (Cortez \& Contreras, 2007).

Como la identificación del SA es difícil debido a los desacuerdos sobre la definición y la falta de instrumentos adecuados que ofrezcan un método preciso y confiable para identificar a las personas con esa condición, el psicólogo estadounidense James Gilliam creó en 2001 la Gilliam Asperger's Disorder Scales (Escala Gilliam para evaluar Trastorno de Asperger, GADS) en su primera versión, con el fin de llenar este vacío (Gilliam, 2008).

Actualmente, existen a nivel mundial siete cuestionarios de detección del SA específicos para niños, y seis para adultos (Attwood, 2009). Dentro de éstos, la Gilliam Asperger's Disorder Scale (GADS), es la que abarca el mayor rango de edad y la que ha demostrado 
tener buenas propiedades psicométricas, siendo sus resultados efectivos para identificar a las personas con SA (Gilliam, 2008; Woodbury-Smith \& Volkmar, 2009) y además, basándose en los criterios diagnósticos actuales (Gilliam, 2008).

$\mathrm{Al}$ observar en detalle las características anteriores, es posible señalar que la GADS es una escala de calificación conductual, cuyo propósito es ayudar a que los profesionales diagnostiquen Síndrome de Asperger (Gilliam, 2008). Esta escala se utiliza para evaluar sujetos de los 3 a los 22 años de edad, por lo que cubre un rango más amplio que otros instrumentos, y que en estudios posteriores se ha comprobado su precisión diagnóstica en este rango (Mayes, Calhoun, Murray, Morrow, Yurich, Mahr, Cothren, Purichia, Bouder \& Petersen, 2009). En relación a la aplicación, cualquiera que tenga contacto directo y sostenido con la persona con SA (padres, familiares, profesores, etc.) puede administrar el instrumento. Además, los ítems de la GADS están basados en los criterios diagnósticos para Trastorno de Asperger publicados en el Manual Diagnóstico y Estadístico de los Trastornos Mentales, cuarta versión revisada (DSM-IV-TR), la Clasificación Internacional de los Trastornos Mentales y del Comportamiento, décima edición (CIE-10) e investigaciones actuales (Gilliam, 2008).

La última versión de la GADS corresponde al año 2008 (Gilliam, 2008). En Chile, sólo se dispone de la GADS traducido al español en México por la licenciada María Guerra, de la Universidad Autónoma de México. Pese a ello, cabe señalar que si bien este instrumento fue traducido al español en dicho país, no fue validado ni estandarizado, por lo que sólo se cuenta con las normas de Estados Unidos (Gilliam, 2008).

Es muy importante contar con un instrumento validado para la detección del SA, debido a que la detección temprana facilita la intervención apropiada que puede mejorar algunos aspectos de los déficits que presentan las personas con SA (como mejorar las conductas sociales, mejorar el afrontamiento de las dificultades, etc.), ayudando así a la posible integración (Ruiz-Lázaro, Posada \& Hijano, 2009). Además, puede disminuir las secuelas secundarias de los problemas en la comunicación y la interacción social (Cortez \& Contreras, 2007), y como la GADS demostró tener confiabilidad y validez en la muestra normativa (Gilliam, 2008), es que esta investigación intenta responder si esta escala conserva las propiedades psicométricas de la muestra normativa al aplicarla en la población chilena con Síndrome de Asperger.

\section{Método}

\section{Participantes}

Se trabajó con una muestra no probabilística intencional, en dos fases: una fase piloto y una fase final. La muestra en la fase piloto, estuvo compuesta por 36 sujetos de diversas regiones de Chile, de los cuales 31 corresponden a hombres $(86,1 \%)$ y 5 a mujeres $(13,9 \%)$. Las edades comprendidas fueron entre $5 \mathrm{y}$ 22 años (media $=12.06 ;$ D.E. $=5.20$ ).

La fase final estuvo compuesta por 111 sujetos, con representación de todas las regiones del país, de los cuales 100 corresponden a hombres $(90,1 \%)$ y 11 a mujeres $(9,9 \%)$. Las edades comprendidas fueron entre 3 y 22 años de edad (media $=12.12 ; D . E .=5.22$ ). El grado de parentesco o vínculo que tuvieron las personas que respondieron el cuestionario con los sujetos con $\mathrm{SA}$, fueron principalmente madres (82.9\%).

\section{Instrumento}

\section{Escala Gilliam para evaluar Trastorno de Asperger (GADS)}

La GADS está diseñada para ser utilizada en sujetos que presentan Síndrome de Asperger entre 3 y 22 años de edad. Es un instrumento autoadministrado por personas que tengan contacto regular con el sujeto que presenta síndrome de Asperger. El cuestionario consta de 32 ítems tipo likert, divididos en cuatro subescalas que describen conductas específicas, observables y medibles. Cada subescala consta de ítems que describen conductas características del síndrome de Asperger. Los ítems 1 a 10 componen la subescala de Interacción Social y describen las conductas sociales interactivas, las expresiones de intentos comunicativos y las conductas cognitivas y emocionales. Los ítems 11 a 18, corresponden a la subescala de Patrones Restringidos de Conducta, describen los patrones dirigidos y estereotipados de conducta que caracterizan al Síndrome de Asperger. La subescala de Patrones Cognitivos, incluye los ítems 19 a 25, los que evalúan habla, lenguaje y habilidades cognitivas. Por último, la subescala de Habilidades Pragmáticas, contiene los ítems 26 a 32, los que hacen referencia a la habilidad del sujeto para comprender y usar el lenguaje en contextos sociales (Gilliam, 2008).

Este instrumento fue normalizado en una muestra de 371 sujetos de 3 a 22 años de edad diagnosticados 
con SA. Los individuos de la muestra normativa provenían de Estados Unidos, Canadá, Gran Bretaña, México, Australia y otros países. La consistencia interna de la GADS fue determinada usando el análisis de Alpha de Cronbach, donde los estudios revelaron coeficientes alpha de 0,88 para Interacción Social; 0,81 para Patrones Restringidos de Conducta; 0,86 para Patrones Cognitivos; 0,84 para Habilidades Pragmáticas; y 0,95 para el Coeficiente de Trastorno de Asperger (Gilliam, 2008).

Para obtener los resultados del instrumento, se calcula el puntaje natural para cada una de las cuatro subescalas de la GADS, sumando los puntajes de todos los ítems. Luego, estos puntajes naturales pueden convertirse a rangos percentiles y puntajes estándar derivados con una media de 10 y una desviación estándar de 3. La suma de los puntajes estándar de las subescalas se convierte en el Coeficiente de Trastorno de Asperger y en el percentil asociado. Como el Coeficiente de Trastorno de Asperger incluye todas las evaluaciones de las subescalas, es la mejor medición global para determinar la probabilidad de que la persona tenga Síndrome de Asperger. Si el Coeficiente de Trastorno de Asperger de la persona es de 80 o más, se considera que esa persona tiene SA (Gilliam, 2008).

Tabla 1

Guía para interpretar el Coeficiente de Trastorno de Asperger

\begin{tabular}{cccc}
\hline $\begin{array}{c}\text { Coeficiente de Trastorno de } \\
\text { Asperger }\end{array}$ & $\begin{array}{c}\text { Rango } \\
\text { percentil }\end{array}$ & $\begin{array}{c}\text { Porcentaje de la } \\
\text { muestra normativa }\end{array}$ & $\begin{array}{c}\text { Probabilidad de trastorno } \\
\text { de Asperger }\end{array}$ \\
\hline$\geq 80$ & 9 a 100 & 92 & Alta/probable \\
70 a 79 & 2 a 8 & 7 & Límite \\
$\leq 69$ & 1 & 1 & Baja/no probable \\
\hline
\end{tabular}

\section{Procedimiento}

Para realizar la presente investigación, se realizó la búsqueda de cuestionarios de detección y/o diagnóstico de Síndrome de Asperger. De esta forma se selecciona la GADS. Se verificó que dicho instrumento no estaba adaptado ni validado en nuestro país.

La GADS fue ingresada a un programa de Google Docs e impresa en papel para las personas sin acceso a internet. Se elaboró el consentimiento informado, donde se informa a los participantes que su colaboración es voluntaria, que los datos son confidenciales y que sólo se utilizarían para fines de la presente investigación.

\section{Fase piloto}

Para la obtención de la muestra se contactaron los participantes a través de las redes sociales, en su mayoría integrantes de la Fundación Asperger Chile y de las diferentes agrupaciones que existen en Chile, contacto telefónico, por correo electrónico, y contacto directo asistiendo a las charlas que imparte la Fundación Asperger Chile. A través del programa Google Docs, se traspasó el cuestionario a un formato on-line, para así abarcar todas las regiones. Se adjuntó el link del cuestionario en las páginas de Facebook de la Fundación Asperger Chile, la Agrupación Asperger Antofagasta, Asperger Quinta, Asperger comuna de Tucapel (Bío-Bío), Agrupación de madres de niños con autismo en San Antonio, y además, se creó una página de Facebook especial para poder difundir la información. También se enviaron cuestionarios a través de correo electrónico, previo contacto personal con madres de niños Asperger que participan en la fundación o agrupaciones. Además, se contactaron algunos colegios con integración, que contaban con algún alumno con SA, donde se les envío a los apoderados el cuestionario on-line, mientras que a los que no tenían acceso a internet, se entregó el material en papel para ser completado.

La aplicación de esta fase tuvo por objetivo evaluar aspectos propios de la administración de la prueba, obtener análisis preliminares de la confiabilidad del instrumento y la exploración de la estructura factorial del cuestionario.

Los datos obtenidos on-line se ingresaban automáticamente a una planilla Excel a través de Google Docs, donde luego se trasladaron a una planilla del programa estadístico SPSS (versión 15.0). Los datos reunidos a través de los cuestionarios impresos se ingresaron directamente a la planilla SPSS. Una vez integrados todos los datos se corrigió el cuestionario de acuerdo a 
la corrección planteada por el autor. Posteriormente, se obtuvieron cálculos descriptivos y se realizó el análisis de confiabilidad a través del alpha de Cronbach, y la validez de constructo a través del análisis factorial.

\section{Fase final}

La realización de esta fase tuvo la finalidad de obtener los indicadores psicométricos definitivos de confiabilidad y validez propuestos en los objetivos específicos de esta investigación. La técnica de recolección de datos fue la misma utilizada en la fase piloto.

En los análisis psicométricos, se procedió a estimar la confiabilidad total de la GADS y de cada una de sus subescalas, a través del alpha de Cronbach. Se evaluó la Validez de Constructo, a través del Análisis Factorial Exploratorio.

\section{Resultados}

\section{Fase piloto}

La confiabilidad global de la escala GADS, obtuvo un alfa de Cronbach de 0,92, mientras que las subescalas obtuvieron un alfa de Cronbach entre 0,73 y 0,85 .
La correlación ítem-total de las cuatro subescalas, en todos los casos fue superior a 0,30 , mostrando la homogeneidad de los ítems. Sólo el ítem 1 obtuvo una correlación negativa.

Respecto del análisis factorial exploratorio, los índices de Kaiser-Meyer-Olkin (KMO) y la prueba de esfericidad de Bartlett, confirman la adecuación de la matriz de correlaciones, y por lo tanto, fue procedente llevar a cabo este análisis. El valor de KMO fue de 0,30, mientras que la prueba de Bartlett fue significativa $\left(c h i^{2}=1092.88 ; g l=496 ; p=.00\right)$.

Se realizó el análisis factorial exploratorio por método de componentes principales y rotación Varimax, del cual se tuvo una solución factorial de 9 factores que explican el 80,9\% de la varianza.

\section{Fase final}

Todas las subescalas se calificaron de 0 a 3 . Las medias y las desviaciones estándar de los puntajes naturales y estándar obtenidas en la fase final en cada subescala se indican en la Tabla 2.

La confiabilidad global de la escala GADS, obtuvo un alfa de Cronbach de 0,92 , mientras que las subescalas obtuvieron un alfa de Cronbach entre 0,73 y 0,87 (ver Tabla 2).

Tabla 2:

Medias y desviación estándar de los puntajes de los puntajes naturales y estándar, coeficiente Trastorno de Asperger y Confiabilidad (fase final).

\begin{tabular}{cccccc}
\hline & $\begin{array}{c}\text { Media } \\
\text { Puntajes } \\
\text { Naturales }\end{array}$ & $\begin{array}{c}\text { D.E. } \\
\text { Puntajes } \\
\text { Naturales }\end{array}$ & $\begin{array}{c}\text { Media } \\
\text { Puntajes } \\
\text { Estándar }\end{array}$ & $\begin{array}{c}\text { D.E. } \\
\text { Puntajes } \\
\text { Estándar }\end{array}$ & $\begin{array}{c}\text { Alfa de } \\
\text { Cronbach }\end{array}$ \\
\hline Subescala Interacción Social & 23.68 & 4.05 & 10.49 & 2.63 & .75 \\
Subescala Patrones Rest. de Conducta & 17.80 & 4.61 & 11.79 & 2.98 & .82 \\
Subescala Patrones Cognitivos & 18.43 & 2.82 & 12.41 & 2.00 & .73 \\
Subescala Habilidades Pragmáticas & 17.13 & 4.32 & 12.08 & 3.10 & .87 \\
Sumatoria Puntaje Estándar & & & 46.74 & 8.59 & \\
Coeficiente Síndrome de Asperger & & & 111.25 & 14.30 & \\
Escala Global & & & & & \\
\hline
\end{tabular}


La correlación ítem-total de la Subescala Interacción Social, en la fase final, en todos los casos fue superior a 0,37, a excepción del ítem 8:"Requiere de instrucciones específicas para comenzar sus tareas", mostrando así la homogeneidad de los reactivos $\mathrm{Al}$ igual que en la fase anterior, sólo el ítem 1: "Presta atención a estímulos sociales/medio ambientales", tuvo una correlación negativa. Las medias de los ítems de esta subescala variaron entre 1,26 y 2,64. Respecto de las desviaciones estándar, las variaciones se encontraron entre 0,66 y 0,88 . A continuación, en tabla 3 se representa el análisis de ítems de la primera subescala.

Tabla 3

Medias, Desviación Estándar (D.E.) y correlación ítem total de la Subescala Interacción Social (fase final).

\begin{tabular}{lccc}
\hline & Media & D.E. & $\mathrm{r}_{1+\mathrm{T}}$ \\
\hline Presta atención a estímulos sociales/medio ambientales. & 1.26 & .72 & -.30 \\
Se le dificulta cooperar en un grupo. & 2.53 & .67 & .51 \\
Tiene dificultades para jugar con otros niños. & 2.56 & .70 & .49 \\
Parece desconocer las reglas sociales o los códigos de conducta. & 2.35 & .88 & .72 \\
Carece de empatía (comprensión de la forma en que otros sienten). & 2.23 & .77 & .57 \\
Requiere de seguridad excesiva si las cosas cambian o funcionan mal. & 2.45 & .71 & .53 \\
Le hace falta sutileza en la expresión de las emociones (por ejemplo, muestra angustia o & 2.64 & .70 & .59 \\
afecto de manera desproporcionada ante la situación). & & & \\
Requiere de instrucciones específicas para comenzar sus tareas. & 2.47 & .69 & .29 \\
Expresa sentimientos de frustración o enojo de manera inapropiada. & 2.57 & .75 & .49 \\
Se siente frustrado rápidamente cuando está inseguro de lo que se requiere. & 2.62 & .66 & .37 \\
\hline
\end{tabular}

La correlación ítem-total de la Subescala Patrones Restringidos de Conducta en la fase final, fue en todos los casos superior a 0,39, mostrando la homogeneidad de los ítems. Las medias variaron entre 1,86 y 2,74 .
Respecto de las desviaciones estándar, las variaciones se encontraron entre 0,55 y 1,00. A continuación, en tabla 4 se representa el análisis de ítems de la segunda subescala.

Tabla 4

Medias, Desviación Estándar (D.E.) y correlación ítem total de la Subescala Patrones Restringidos de Conducta (fase final).

\begin{tabular}{lccc}
\hline & Media & D.E. & $\mathrm{r}_{1+\mathrm{T}}$ \\
\hline Parece no estar entusiasmada o estar infeliz cuando la entretienen o es alabada. & 1.86 & .91 & .50 \\
Desconoce o es insensible a las necesidades de los demás. & 2.16 & .85 & .64 \\
Demuestra formas excéntricas de comportamiento. & 2.44 & .79 & .63 \\
Presenta una preocupación por objetos o temas específicos que es anormal en intensidad & 2.74 & .55 & .40 \\
o concentración. & 2.31 & .80 & .39 \\
Requiere de amplias instrucciones por parte de los demás. & 2.17 & 1.0 & .64 \\
Expresa sentimientos de empatía de manera inapropiada. & .10 & .94 & .65 \\
Muestra movimientos motores gruesos torpes y no coordinados. & 2.10 & 2.02 & .98 \\
Muestra movimientos inusuales o no coordinados cuando camina o corre. & .52 \\
\hline
\end{tabular}


La correlación ítem-total de la Subescala Patrones Cognitivos en la fase final, en todos los casos fue superior a 0,43 , excepto el ítem 24: "Demuestra una excelente memoria", mostrando la homogeneidad de los ítems. Las medias de los ítems de esta subescala variaron entre 2,55 y 2,81 . Respecto de las desviaciones estándar, las variaciones se encontraron entre 0,48 y 0,75 . A continuación, en la Tabla 5, se representa el análisis de ítems de la tercera subescala.

Tabla 5

Medias, Desviación Estándar (D.E.) y correlación ítem total de la Subescala Patrones Cognitivos (fase final).

\begin{tabular}{lccc}
\hline & Media & D.E. & r ${ }_{1+\mathrm{T}}$ \\
\hline Habla sobre un solo tema de manera excesiva. & 2.55 & .72 & .43 \\
Demuestra habilidades o conocimientos superiores en temas o actividades específicas. & 2.70 & .60 & .51 \\
Emplea un lenguaje excesivamente preciso o pretencioso. & 2.50 & .75 & .46 \\
Otorga significados muy concretos a las palabras. & 2.65 & .67 & .51 \\
Tiene dificultades para entender el humor o las bromas. & 2.61 & .69 & .46 \\
Demuestra una excelente memoria. & 2.60 & .64 & .23 \\
Muestra un interés intenso y obsesivo en ciertos temas. & 2.81 & .48 & .54 \\
\hline
\end{tabular}

La correlación ítem-total de la Subescala Habilidades Pragmáticas en la fase final, en todos los casos fue superior a 0,33 , mostrando la homogeneidad de los ítems. Las medias de los ítems de esta subescala variaron entre 2,40 y 2,49. Respecto de las desviaciones estándar, las variaciones se encontraron entre 0,74 y 0,91. A continuación, en la Tabla 6 , se representa el análisis de ítems de la cuarta subescala.

Tabla 6

Medias, Desviación Estándar (D.E.) y correlación ítem total de la Subescala Habilidades Pragmáticas (fase final).

\begin{tabular}{|c|c|c|c|}
\hline & Media & D.E. & $\mathrm{r}_{1+\mathrm{T}}$ \\
\hline Tiene dificultades para entender expresiones de uso común. & 2.49 & .74 & .72 \\
\hline Tiene dificultades para identificar cuando alguien se está burlando. & 2.45 & .82 & .79 \\
\hline $\begin{array}{l}\text { Tiene dificultades para entender cuando él o ella está siendo ridiculizado, expuesto o cuando } \\
\text { se están divirtiendo a costa suya. }\end{array}$ & 2.42 & .82 & .76 \\
\hline Tiene dificultades para entender el motivo por el cual él o ella no le agrada a alguien. & 2.46 & .84 & .73 \\
\hline No predice probables consecuencias en situaciones sociales. & 2.49 & .79 & .67 \\
\hline Tiene problemas para hacer creer algo o fingir. & 2.42 & .91 & .58 \\
\hline Cuando está confundida, no pide una aclaración, pero cambia a un tema conocido. & 2.40 & .86 & .33 \\
\hline
\end{tabular}

Se realizó un análisis factorial exploratorio forzando la solución de cuatro factores propuesto en la escala original. Los resultados obtenidos, arrojaron que los índices de Kaiser-Meyer-Olkin (KMO) y la prueba de esfericidad de Bartlett confirman la adecuación de la matriz de correlaciones, y por lo tanto, fue procedente llevar a cabo este análisis. El valor de KMO fue de 0,83, mientras que la prueba de Bartlett fue significativa $\left(c h i^{2}=2114.68 ; g l=496 ; p=.00\right)$.

Se realizó el análisis factorial exploratorio por método de componentes principales y rotación Varimax (Tabla 7), del cual se obtuvo una solución factorial de 8 factores que explican el 69,99\% de la varianza. 
El primer factor explica el 19,04\% de la varianza y contiene los ítems 27, 28, 23, 29, 22, 26, 30 y 31, cuyas cargas factoriales varían entre 0,61 y 0,88 . El segundo factor explica el $8,78 \%$ de la varianza y contiene a los ítems 7, 10, 9 y 6, variando las cargas factoriales entre 0,56 y 0,77 . El tercer factor explica el $8,23 \%$ de la varianza, contiene los ítems 5, 16, 32, 4 y 13 y la carga factorial varía entre 0,48 y 0,69 . El cuarto factor explica el 7,79\% de la varianza y contiene a los ítems 3,2 y 21 , mientras que sus cargas factoriales varían entre 0,51 y 0,75 . El quinto factor explica el $7,54 \%$ de la varianza, contiene a los ítems 18,17 y 19 y sus cargas factoriales varían entre 0,49 y 0,88 . El sexto factor explica el 7,38\% de la varianza, contiene los ítems $8,14,15,1$ y 25 , variando las cargas factoriales entre $-0,51$ y 0,72 . El séptimo factor explica el $6,83 \%$ de la varianza, contiene a los ítems 20 y 24 y sus cargas factoriales varían entre 0,77 y 0,79 . El octavo y último factor explica 4,39\% de la varianza y contiene los ítems 11 y 12 , variando su carga factorial entre 0,45 y 0,66 .

Tabla 7

Análisis de componentes principales y rotación Varimax de la GADS (fase final)

\begin{tabular}{|c|c|c|c|c|c|c|c|c|}
\hline & 1 & 2 & 3 & 4 & 5 & 6 & 7 & 8 \\
\hline 27. Tiene dificultades para identificar cuando alguien se está burlando. & .88 & & & & & & & \\
\hline $\begin{array}{l}\text { 28. Tiene dificultades para entender cuando él o ella está siendo ridiculizado, expuesto o } \\
\text { cuando se están divirtiendo a costa suya. }\end{array}$ & & & & & & & & \\
\hline 23. Tiene dificultades para entender el humor o las bromas. & .83 & & & & & & & \\
\hline 29. Tiene dificultades para entender el motivo por el cual él o ella no le agrada a alguien. & .80 & & & & & & & \\
\hline 22. Otorga significados muy concretos a las palabras. & .73 & & & & & & & \\
\hline 26. Tiene dificultades para entender expresiones de uso común. & .73 & & & & & & & \\
\hline 30. No predice probables consecuencias en situaciones sociales. & .67 & & & & & & & \\
\hline 31. Tiene problemas para hacer creer algo o fingir. & .61 & & & & & & & \\
\hline 7. Le hace falta sutileza en la expresión de las emociones. & & .77 & & & & & & \\
\hline 10. Se siente frustrado rápidamente cuando está inseguro de lo que se requiere. & & .74 & & & & & & \\
\hline 9. Expresa sentimientos de frustración o enojo de manera inapropiada. & & .66 & & & & & & \\
\hline 6. Requiere de seguridad excesiva si las cosas cambian o funcionan mal. & & .56 & & & & & & \\
\hline 5. Carece de empatía (comprensión de la forma en que otros sienten). & & & 69 & & & & & \\
\hline 16. Expresa sentimientos de empatía de manera inapropiada. & & & .58 & & & & & \\
\hline 32. Cuando está confundida, no pide una aclaración, pero cambia a un tema conocido. & & & .58 & & & & & \\
\hline 4. Parece desconocer las reglas sociales o los códigos de conducta. & & & .52 & & & & & \\
\hline 13. Demuestra formas excéntricas de comportamiento. & & & .48 & & & & & \\
\hline 3. Tiene dificultades para jugar con otros niños. & & & & .75 & & & & \\
\hline 2. Se le dificulta cooperar en grupo. & & & & .72 & & & & \\
\hline 21. Emplea un lenguaje excesivamente preciso o pretencioso. & & & & .51 & & & & \\
\hline 18. Muestra movimientos inusuales o no coordinados cuando camina o corre. & & & & & .88 & & & \\
\hline 17. Muestra movimientos motores gruesos torpes y no coordinados. & & & & & .83 & & & \\
\hline 19. Habla sobre un solo tema de manera excesiva & & & & & .49 & & & \\
\hline 8. Requiere de instrucciones específicas para comenzar sus tareas. & & & & & & .72 & & \\
\hline $\begin{array}{l}\text { 14. Presenta una preocupación por objetos o temas específicos que es anormal en inten- } \\
\text { sidad o concentración. }\end{array}$ & & & & & & .68 & & \\
\hline 15. Requiere de amplias instrucciones por parte de los demás. & & & & & & 61 & & \\
\hline 1. Presta atención a estímulos sociales/medio ambientales. & & & & & & -.51 & & \\
\hline 25. Muestra un interés intenso y obsesivo en ciertos temas & & & & & & .46 & & \\
\hline 20. Demuestra habilidades o conocimientos superiores en temas o actividades específicas. & & & & & & & .79 & \\
\hline 24. Demuestra una excelente memoria. & & & & & & & .77 & \\
\hline 11. Parece no estar entusiasmada o estar infeliz cuando la entretienen o es alabada. & & & & & & & & .66 \\
\hline 12. Desconoce o es insensible a las necesidades de los demás. & & & & & & & & .45 \\
\hline
\end{tabular}


Se realizó el análisis factorial exploratorio por método de componentes principales y rotación Varimax (Tabla 8). La solución factorial de 4 factores explica el $53,38 \%$ de la varianza.

El primer factor explica el $20,4 \%$ de la varianza y contiene los ítems $27,23,28,29,26,30,22,31$, 13 y 21 , cuyas cargas factoriales varían entre 0,46 y 0,86 . El segundo factor explica el $13,2 \%$ de la varianza y contiene a los ítems $9,7,10,17,16$, $18,4,6$ y 11 , variando las cargas factoriales entre 0,43 y 0,78 . El tercer factor explica el $12,42 \%$ de la varianza y contiene los ítems $2,3,15,8,14,5$, 12 y 1 , cuyas cargas factoriales varían entre $-0,35$ y 0,69 . El cuarto y último factor, explica el 7,36\% de la varianza y contiene los ítems 20,24, 25, 19 y 32, variando las cargas factoriales entre -0.37 y 0,79 .

Tabla 8

Análisis de componentes principales y rotación Varimax de la GADS forzando cuatro factores.

\begin{tabular}{|c|c|c|c|c|}
\hline & $\begin{array}{c}\text { Factor } \\
1\end{array}$ & $\begin{array}{c}\text { Factor } \\
2\end{array}$ & $\begin{array}{c}\text { Factor } \\
3 \\
\end{array}$ & $\begin{array}{c}\text { Factor } \\
4\end{array}$ \\
\hline 27. Tiene dificultad para identificar cuando alguien se está burlando & .86 & & & \\
\hline 23. Tiene dificultades para entender el humor o las bromas & .85 & & & \\
\hline $\begin{array}{l}\text { 28. Tiene dificultades para entender cuando él o ella está siendo ridiculizado, expuesto o cuando } \\
\text { se están divirtiendo a costa suya }\end{array}$ & .84 & & & \\
\hline 29. Tiene dificultades para entender el motivo por el cual él o ella no le agrada a alguien & .77 & & & \\
\hline 26. Tiene dificultades para entender expresiones de uso común & .75 & & & \\
\hline 30. No predice probables consecuencia en situaciones sociales & .72 & & & \\
\hline 22. Otorga significados muy concretos a las palabras & .70 & & & \\
\hline 31. Tiene problemas para hacer creer algo o fingir & .67 & & & \\
\hline 13. Demuestra formas excéntricas de comportamiento & .53 & & & \\
\hline 21. Emplea un lenguaje excesivamente preciso o pretencioso & .46 & & & \\
\hline 9. Expresa sentimientos de frustración o enojo de manera inapropiada & & .78 & & \\
\hline 7. Le hace falta sutileza en la expresión de las emociones & & .68 & & \\
\hline 10. Se siente frustrado rápidamente cuando está inseguro de lo que se requiere & & .67 & & \\
\hline 17. Muestra movimientos motores gruesos torpes y no coordinados & & .58 & & \\
\hline 16. Expresa sentimientos de empatía de manera inapropiada & & .58 & & \\
\hline 18. Muestra movimientos inusuales o no coordinados cuando camina o corre & & .57 & & \\
\hline 4. Parece desconocer las reglas sociales o los códigos de conducta & & .54 & & \\
\hline 6. Requiere de seguridad excesiva si las cosas cambian o funcionan mal & & .50 & & \\
\hline 11. Parece no estar entusiasmada o estar infeliz cuando la entretienen o es alabada & & .43 & & \\
\hline 2. Se le dificulta cooperar en grupo & & & .69 & \\
\hline 3. Tiene dificultades para jugar con otros niños & & & .68 & \\
\hline 15. Requiere de amplias instrucciones por parte de los demás & & & .66 & \\
\hline 8. Requiere de instrucciones específicas para comenzar sus tareas & & & .62 & \\
\hline $\begin{array}{l}\text { 14. Presenta una preocupación por objetos o temas específicos que es anormal en intensidad } \\
\text { o concentración }\end{array}$ & & & .58 & \\
\hline 5. Carece de empatía & & & .54 & \\
\hline 12. Desconoce o es insensible a las necesidades de los demás & & & .45 & \\
\hline 1. Presta atención a estímulos sociales/medio ambientales & & & -.35 & \\
\hline 20. Demuestra habilidades o conocimientos superiores en temas o actividades específicas & & & & .79 \\
\hline 24. Demuestra una excelente memoria & & & & .60 \\
\hline 25. Muestra un interés intenso y obsesivo en ciertos temas & & & & .57 \\
\hline 19. Habla sobre un solo tema de manera excesiva & & & & .49 \\
\hline 32. Cuando está confundida no pide una aclaración, pero cambia a un tema conocido & & & & -.37 \\
\hline
\end{tabular}




\section{Discusión y conclusiones}

Cada vez existe mayor conciencia de la necesidad de diagnosticar el Síndrome de Asperger en una edad temprana, ya que esto posibilita la pronta intervención en estos niños, mejorando algunos aspectos de los déficits que presentan y ayudando así a su integración.

Durante la etapa escolar, el conocimiento por parte de los profesores y compañeros acerca del déficit del desarrollo social del niño, permite facilitar la adaptación de las demandas sociales y expectativas comportamentales a su perfil particular de competencias cognitivas y sociales, evitando así exigencias que el niño no podrá cumplir, y que le generarían otros problemas como frustración, ansiedad, problemas conductuales, etc. Además, permite la planificación e implementación de estrategias y programas de intervención especializados para trabajar las habilidades sociales de estos niños. Por otra parte, en la adolescencia y en la edad adulta, el diagnóstico permite que el joven conozca la naturaleza de su déficit social y sus implicancias, lo que le brinda una oportunidad para comprender el origen de su problema y dejar de sentirse diferente a los demás sin saber por qué (Martín, 2008).

Es por esto, la importancia de contar con un instrumento adaptado a nuestra población, que cuente con propiedades psicométricas y que ayude en este diagnóstico: cabe señalar, que cualquier instrumento diagnóstico es un apoyo necesario y objetivo de la evaluación, pero que la entrevista a los padres o cuidadores y la observación, también son parte fundamental para completar el diagnóstico.

A continuación, se discutirán los resultados obtenidos a partir de cada fase realizada.

\section{Fase piloto}

La estimación de la confiabilidad interna de las subescalas fueron satisfactorias y en la escala global su confiabilidad se considera alta $(\alpha=0,92)$, considerándose muy satisfactoria (Gilliam, 2008).

La correlación ítem-total no sugirió la eliminación de ítems que pudieran influir significativamente, por lo tanto se decidió aplicar el cuestionario con la totalidad de los reactivos.

Respecto del análisis factorial exploratorio, se obtuvo una solución factorial de 9 factores, encontrándose diferencias respecto de la estructura tetrafactorial propuesta por Gilliam (2008). Si bien los cuatro factores establecidos en la teoría se manifiestan, en este primer análisis se agregan otros más y la distribución de los factores podría definirse como: 1) Habilidades Pragmáticas; 2) Patrones Cognitivos; 3) Interacción Social; 4) Destrezas; 5) Manejo de las emociones; 6) Intereses Restringidos; 7) Patrones Restringidos de Conducta; 8) Estímulos Sociales y 9) Conocimientos Especificos.

En este primer estudio, la GADS mostró propiedades psicométricas adecuadas que permitieron la aplicación de una muestra final y no se reportaron problemas con la comprensión de los ítems.

\section{Fase final}

La estimación de la confiabilidad interna de las subescalas de la GADS fue similar a la de la fase piloto y la escala global obtuvo una consistencia interna igual a la de la primera fase $(\alpha=0,92)$, considerándose muy satisfactoria (Hair, Anderson, Tatham y Black, 1999).

$\mathrm{Al}$ igual que en la fase piloto, la correlación ítemtotal no sugirió la eliminación de ítems que pudieran influir significativamente.

Respecto del análisis factorial exploratorio, a diferencia de la fase piloto donde se obtuvo una solución factorial de 9 factores, en esta fase se obtuvo una solución factorial de 8 factores, encontrándose nuevamente diferencias respecto de la estructura tetrafactorial propuesta por Gilliam (2008). Si bien los cuatro factores establecidos en la teoría se manifiestan, en este primer análisis se agregan cuatro más y la distribución de los factores podría definirse como: 1) Habilidades Pragmáticas, 2) Interacción Social, 3) Relaciones Interpersonales, 4) Interacción con sus Pares, 5) Destrezas, 6) Estímulos Sociales, 7) Patrones Cognitivos y 8) Patrones Restringidos de Conducta.

El primer factor, que corresponde a Habilidades Pragmáticas, hace referencia a la habilidad del sujeto para comprender y usar el lenguaje en contextos sociales. El segundo factor, denominado Interacción Social, describe las conductas sociales interactivas. El tercer factor que corresponde a Relaciones Interpersonales, se refiere a la forma que tiene el sujeto para relacionarse con otras personas. El cuarto factor, que corresponde a Interacción con sus pares, hace referencia a la forma de actuar del sujeto frente a sus pares. El quinto factor, que corresponde a Destrezas, alude a las habilidades de los sujetos. El sexto factor que corresponde a Estímulos Sociales, caracteriza la forma de actuar frente a estímulos externos.

El séptimo factor, que corresponde a Patrones Cognitivos, evalúa lenguaje y habilidades cognitivas. El octavo y último factor, que corresponde a Patrones 
Restringidos de Conducta, hace referencia a patrones de intereses y conductas restringidas y repetitivas.

También se realizó un análisis factorial forzando la estructura de cuatro factores propuesta por Gilliam (2008), y pese a que se pueden identificar las cuatros subescalas, la distribución de algunos ítems varían debido a la amplitud del reactivo, no obstante, ninguno produjo problemas al aplicarlo, ni dificultad en la comprensión. Solamente el ítem 1 "Presta atención a estímulos sociales/medio ambientales", presenta una puntuación inversa, donde su carga factorial es negativa al igual que la correlación ítem-total, lo que podría causar algún tipo de confusión. Por ello, se sugiere que para próximas investigaciones este ítem sea reformulado o eliminado de la subescala y así obtener nuevos resultados que permitan mejorarla.

En cuanto a las subescalas, Habilidades Pragmáticas fue la que sufrió menos modificaciones en sus ítems, lo que la hace confiable para diferenciar problemas únicos en esta área, es decir, problemas a nivel de lenguaje, como por ejemplo, el trastorno semántico-pragmático del lenguaje (Martín-Borreguero, 2008). Pese a que podrían presentarse algunos problemas en la identificación de problemas únicos en las otras subescalas debido a la amplitud de algunos ítems, esto no afectaría el resultado final, es decir, la obtención del Coeficiente de Trastorno de Asperger.

En la adaptación realizada con muestra chilena, no se confirma la estructura tetrafactorial de la escala original, sin embargo, se proporciona una nueva organización factorial de ocho factores, los que no se alejan completamente de estas dimensiones, no distando ninguno de la definición y características del SA propuestas en el DSM-IV-TR y CIE-10, motivo por el que la escala no debería ser afectada.

Debido a la similitud de los resultados obtenidos de la muestra chilena con la muestra normativa, no es necesario ajustar ni modificar las normas y baremos para nuestra población, siendo adecuada la interpretación del coeficiente de Trastorno de Asperger. Esto se sustenta en que el autor de la GADS utilizó una muestra que abarcó diversos países, lo que hace que sea una muestra intercultural.

Los resultados y análisis de esta investigación, permiten concluir que la GADS cuenta con propiedades psicométricas adecuadas, lo que significa que es un instrumento confiable y válido para ser utilizado en la población chilena.

En relación a la edad de diagnóstico, en nuestro país se diagnostica aproximadamente a $\operatorname{los} 9$ años $(D . E .=4.37)$, lo que estaría dentro de la media del diagnóstico a nivel mundial (Attwood, 2009), pero que a la vez nos muestra lo tardío del diagnóstico, dificultando así la posibilidad de intervención en los niños más pequeños.

Dentro de los profesionales que diagnosticaron el síndrome, no aparecieron los pediatras, lo que concuerda con la investigación de Skellern et al. (2005; citado en Cortez \& Contreras, 2007) donde señalan que éstos serían los menos entrenados para el diagnóstico de estos trastornos, siendo los más preparados los neurólogos y psicólogos.

Como lo señala Fernández (2011), son las asociaciones de Asperger las que contribuyen de manera activa a informar a la población y a los profesionales sobre este síndrome, puesto que en ocasiones no se diagnostica correctamente o sólo se diagnostican sus síntomas más evidentes. Es por ello la relevancia de estas asociaciones, las que principalmente se componen por madres, que en forma frecuente se reúnen con el fin de capacitarse y aportar información sobre este síndrome y así poder ayudar a otras personas que comparten con algún sujeto con SA. La participación activa y comprometida de estas mujeres, se ven reflejadas en la colaboración con esta investigación, ya que el 91,7\% de las personas que respondieron el cuestionario, corresponde a madres, sin dejar de mencionar la participación, aunque menos representativa, de padres y profesores.

Debido a las dificultades diagnósticas, muchas personas con SA son mal diagnosticadas, y otras (aunque menos frecuentemente), pueden llevar la etiqueta de Asperger sin serlo, como podrían ser las dos personas del estudio que no cumplen con el puntaje mínimo del Coeficiente de Trastorno de Asperger. Es importante señalar además, que muchos especialistas diagnostican a personas con SA como autista, debido al solapamiento clínico del SA con el autismo, lo que ha planteado de forma ineludible cuestiones pertinentes con respecto a su naturaleza y estatus nosológico, desencadenando un debate controvertido sobre la validez del síndrome como categoría diagnóstica independiente y distintiva (Martín-Borreguero, 2008).

Es por esto, que el DSM-V lo eliminó como una categoría independiente, y lo incluyó dentro del Espectro Autista, lo que ha generado una gran controversia. Tal vez, hubiera sido mejor, capacitar a los profesionales en los TGD, con el fin de que puedan diagnosticar y diferenciar un trastorno de otro, y no de reunirlos todos bajo un mismo nombre con el fin de evitar ser mal diagnosticados. Pese a esto, el CIE-10, mantendrá el Síndrome de Asperger como una categoría independiente, motivo por el que los niños seguirán siendo diagnosticados bajo esta categoría, ya que el sistema de Salud Pública y el 
Ministerio de Educación, trabajan con estos criterios diagnósticos (CIE-10).

Poder diferenciar a una persona autista de un Asperger es relevante, ya que el autismo conlleva una etiqueta negativa en cuanto a sus déficit, especialmente en lo cognitivo y en el lenguaje, lo que hace aún más difícil su integración en un colegio normal. El niño es aún más discriminado y segregado de sus pares, perjudicando su desarrollo. Al diagnosticar a todos bajo una misma etiqueta, se le está quitando la posibilidad a un niño con Asperger integrarse de una manera más adecuada a la sociedad.

En cuanto a las limitaciones de este estudio, cabe señalar, que pese a que uno de los criterios de inclusión era que los participantes tuvieran un diagnóstico previo de Síndrome de Asperger, esto no se pudo comprobar, ya que las respuestas fueron ingresadas a través de la web, por el difícil acceso de contactar personalmente a los participantes. Como es una muestra específica, se hizo de esta manera para poder abarcar todas las regiones de Chile y poder obtener una muestra de tamaño suficiente. Según algunos autores (Campbell, 2005; Mayes et al., 2009; Toth \& King, 2008), todas las escalas han sido normalizadas sin comprobar el diagnóstico, por lo que sería relevante, que en futuras investigaciones, se pudiera comprobar el diagnóstico de los participantes, para conocer si existen diferencias significativas en los resultados.

Por otro lado, Gilliam (2008), señala que la GADS no solo es útil en el diagnóstico del SA, sino que también en identificar personas con problemas conductuales únicos en las áreas de interacción social, patrones restringidos de conducta, patrones cognitivos y habilidades pragmáticas, y como cada subescala es independiente, se pueden identificar si existen problemas en alguna área aunque la persona no tenga SA. No obstante, otra de las limitaciones de esta investigación, es que se presentaron dificultades en la identificación de problemas conductuales únicos, ya que como se mencionó anteriormente, a excepción de la subescala Habilidades Pragmáticas, los ítems de las otras subescalas fueron redistribuidos en otras dimensiones diferentes a la que propone el autor (Gilliam, 2008), por lo que quizás no sería confiable un diagnóstico de un problema conductual único basado sólo en el puntaje obtenido en una sola dimensión. Sin embargo, esto no influye en el resultado final, es decir, en el Coeficiente de Trastorno de Asperger que es el que nos indica la probabilidad de que la persona tenga SA.

Por lo tanto, como se presentó al inicio de esta investigación, contar con instrumentos válidos y confiables para el diagnóstico del SA es de suma relevancia. Pese a que en los últimos años, los campos de la pediatría, la psiquiatría y la psicología han presenciado un auge notable en el interés de los profesionales clínicos y de la educación por el estudio del SA, no parece haberse traducido en avances significativos en relación al diagnóstico temprano del SA, sino que en general este continúa basándose en el juicio subjetivo que el profesional realiza (Martín-Borreguero, 2008). La GADS en cambio, proporciona datos en forma de puntajes objetivos para determinar qué personas presentan el SA, lo que facilita el diagnóstico, por lo que presenta gran utilidad en la práctica clínica (Gilliam, 2008).

En conclusión, contamos con un instrumento que es breve y fácil de aplicar, que además cuenta con las propiedades psicométricas adecuadas y que en diversos estudios ha demostrado ser eficaz en el diagnóstico de SA, correspondiendo la precisión diagnóstica en un 94\% (Campbell, 2005; Mayes et al., 2009; Toth \& King, 2008), donde el posible error diagnóstico es muy bajo.

\section{Referencias}

Alonso, N., Belinchón, M., Blanco, R., Cáceres, de Diego, M., D., Frías, C., Frontera, M., Giménez, A., Martínez, M., Murillo, E., Nieto, C., \& Saldaña, E. (2009). Hacia un modelo de Apoyos Universitarios a Estudiantes con Síndrome de Asperger: Necesidades y Propuestas. Madrid: Centro de Psicología Aplicada, Universidad Autónoma de Madrid.

Asociación Americana de Psiquiatría (2000). Manual Diagnóstico y Estadístico de los Trastornos Mentales (DSM-IV TR). Madrid: Masson.

Attwood, T. (2009). Guía del Síndrome de Asperger. Barcelona: Paidós.

Caballero, R. (2008). Síndrome de Asperger. En Vázquez, C. y Martínez M. (2008). Los trastornos generales del desarrollo. Una aproximación desde la práctica. Volumen 2: El Síndrome de Asperger (pp. 6-13). Sevilla: Consejería de Educación Junta de Andalucía.

Campbell, J. (2005). Diagnostic Assement of Asperger's Disorder: A Review of Five Third-Party Rating Scales. Journal of Autism and Developmental Disorders, 35, 25-35.

Cererols, R. (2010). Descubrir el Asperger. Valencia: Psylicom Ediciones.

Cortez, M. \& Contreras, M. (2007). Diagnóstico precoz de los trastornos del espectro autista en edad temprana (18-36 meses). Archivos Argentinos de Pediatría, 105, 418-426.

Fernández, A. (2011). ¿Cómo se integra un niño con síndrome de Asperger en un aula ordinaria? Innovación y Experiencias Educativas, 39, 1-12.

Fernández, F. (2010). Orientaciones para el área de educación física sobre alumnos con síndrome de Asperger. EmásF: Revista Digital de Educación Física, 1(3), 56-68. 
Fernández-Jaén, A., Fernández-Mayoralas, D., Calleja-Pérez, B. \& Muñoz, N. (2007). Síndrome de Asperger: diagnóstico y tratamiento. Revista de Neurología, 44, 53-55.

Foley, M. \& Assouline, S. (2010). Atendiendo a las necesidades de estudiantes talentosos con trastornos del espectro de autismo: aproximaciones diagnósticas, terapéuticas y psicoeducativas. Psicoperspectivas, 9, 202-223.

Ghaziuddin, M. (2008). Defining the Behavioral Phenotype of Asperger Syndrome. Journal Autism Developmental Disorders, $38,138-142$.

Gilliam, J. (2008). Escala Gilliam para evaluar Trastorno de Asperger: GADS. México: Manual Moderno.

Hair, J., Anderson, R., Tatham, R. \& Black, W. (1999). Análisis Multivariable ( ${ }^{\mathrm{a}}$ Ed.). Madrid: Prentice Hall.

López-Escobar, M. (2008). Detección, diagnóstico y evaluación de los alumnos con síndrome de Asperger. En Vázquez, C. y Martínez M. (2008). Los trastornos generales del desarrollo. Una aproximación desde la práctica. Volumen 2: El síndrome de Asperger. Sevilla: Consejería de Educación Junta de Andalucía.

Marker, C., Weeks, M. \& Kraegel, I. (2007). Integrating Faith and Treatment for Children with High Functioning Autism Spectrum Disorders. Journal of Psychology and Christianity, 26, 112-121.

Martín-Borreguero, P. (2008). El síndrome de Asperger ¿Excentricidad o discapacidad social? Madrid: Alianza Editorial.

Martínez, E. (2009). Afecta la socialización, no la inteligencia. Medicina y Salud, 1. Recuperado de http://vlex.com/vid/afectasocialización-no-inteligencia-77616772

Mayes, S., Calhoun, S., Murray, M., Morrow, J., Yurich, K., Mahr, F., Cothren, S., Purichia, H., Bouder, J. \& Petersen, C. (2009). Comparison of Scores on the Checklist for Autism Spectrum Disorder, Childhood Autism Rating Scale, and Gilliam Asperger's Disorder Scale for Children with Low Functioning Autism, High
Functioning Autism, Asperger's Disorder, ADHD, and Typical Development. Journal of Autism and Developmental Disorders, 39, 1682-1693.

McPartland, J. \& Volkmar, F. (2009). Current Clinical Practice in Asperger Disorder. Psychiatric Times, 26, 52-56.

Mella, S., Araneda, K., Ormazábal, A., Salinas, N. \& Sepúlveda, R. (2011). Desempeño Ocupacional Escolar de Jóvenes con Síndrome de Asperger que asisten al Sistema de Educación Regular. Revista Chilena de Terapia Ocupacional, 11, 84-101.

Organización Mundial de la Salud (2000). Clasificación de los trastornos mentales y del comportamiento, $10^{\circ}$ edición (CIE-10). Madrid: Panamericana.

Pacheco, B., Sandoval, C. \& Torrealba, C. (2008). Episodio depresivo en adolescente con Trastorno de Asperger: Reporte de un caso. Revista chilena de Neuro-Psiquiatría, 46, 199-206.

Ramírez, C. (2010). ¿Conocemos el Síndrome de Asperger? Revista digital para profesionales de la enseñanza, 9. Recuperado de http://www2.fe.ccoo.es/andalucia/docupdf.aspx?d=7388\&s=

Rodríguez, F. \& Ridao, S. (2011). La docencia virtual en el tratamiento comunicativo de enfermedades raras: Descripción de un curso destinado a profesionales en síndrome de Asperger. Revista de Educación a Distancia - Docencia Universitaria en la Sociedad del Conocimiento, 3. Recuperado de http://www.um.es/ead/ reddusc/3/ridao_munoz.pdf

Ruiz-Lázaro, P., Posada, M. \& Hijano, F. (2009). Trastornos del espectro autista. Detección precoz, herramientas de cribado. Revista Pediatría de Atención Primaria, 11, 381-397.

Toth, K. \& King, B. (2008). Asperger's Syndrome: Diagnosis and Treatment. The American Journal of Psychiatry, 165, 958-963.

Villanueva, M. (2011). Tiene un estilo peculiar. Innovación y Experiencias Educativas, 43. Recuperado de http://www.csicsif.es/andalucia/modules/mod_ense/revista/pdf/Numero_43/ MARIA\%20VILLANUEVA\%20GARCIA_1.pdf

Woodbury-Smith, M. \& Volkmar, F. (2009). Asperger Syndrome. European Child Adolescent Psychiatry, 18, 2-11. 


\section{Anexos \\ ESCALA GILLIAM PARA EVALUAR TRASTORNO DE ASPERGER (GADS)}

Instrucciones: valore cada uno de las afirmaciones de acuerdo a la frecuencia en que ocurren. Utilice las siguientes pautas para sus puntuaciones:

0 Nunca observado: Nunca ha visto que la persona se comporte de esa manera.

1 Rara vez observado: La persona se comporta de esa manera de 1 a 2 veces por cada periodo de 6 horas.

2 Algunas veces observado: La persona se comporta de esa manera de 3 a 4 veces por cada periodo de 6 horas.

3 Frecuentemente observado: La persona se comporta de esa manera al menos 5 veces por cada período de 6 horas.

Utilizando la escala de 0 a 3 que aparece junto a cada enunciado, califique su impresión de la frecuencia con que la persona demuestra el comportamiento. Indique su calificación encerrando en un círculo en el número apropiado. Base su calificación en su conocimiento y experiencia directa con la persona con la cual ha convivido. Recuerde calificar cada enunciado. Si no está seguro acerca de cómo calificar alguno de ellos, posponga su calificación y observa a la persona durante un período de 6 horas para determinar su calificación.

\section{Subescala de Interacción Social}

La persona:

$\begin{array}{cccc}\begin{array}{c}\text { Nunca } \\ \text { observado }\end{array} & \begin{array}{c}\text { Raras veces } \\ \text { observado }\end{array} & \begin{array}{c}\text { Algunas } \\ \text { veces } \\ \text { observado }\end{array} & \begin{array}{c}\text { Frec. } \\ \text { observ. }\end{array} \\ & & \end{array}$

Presta atención a estímulos sociales/medio ambientales

0

Se le dificulta cooperar en un grupo

0

2

3

Tiene dificultades para jugar con otros niños

0

Parece desconocer las reglas sociales o los códigos de conducta

0

Carece de empatía (comprensión de la forma en que otros sienten)

0

Requiere de seguridad excesiva si las cosas cambian o funcionan mal....

0

Le hace falta sutileza en la expresión de las emociones (por ejemplo, muestra angustia o afecto de manera desproporcionada ante la situación)

1
1

2

3

Requiere de instrucciones específicas para comenzar sus tareas

\begin{tabular}{llll}
0 & 1 & 2 & 3 \\
0 & 1 & 2 & 3 \\
0 & 1 & 2 & 3 \\
0 & 1 & 2 & 3 \\
\hline
\end{tabular}




\section{Subescala de Patrones Restringidos de Conducta}

La persona:

\begin{tabular}{ccc}
$\begin{array}{c}\text { Nunca } \\
\text { observado }\end{array}$ observado veces Algunas $\begin{array}{c}\text { Frec. } \\
\text { veces observ. } \\
\text { observado }\end{array}$ & \multicolumn{2}{c}{}
\end{tabular}

Parece no estar entusiasmada o estar infeliz cuando la entretienen o es alabada .....

Desconoce o es insensible a las necesidades de los demás

Demuestra formas excéntricas de comportamiento

0

0

1

23

Presenta una preocupación por objetos o temas específicos que es anormal en intensidad o concentración

.

Requiere de amplias instrucciones por parte de los demás .

Expresa sentimientos de empatía de manera inapropiada .......

Muestra movimientos motores gruesos torpes y no coordinados .

Muestra movimientos inusuales o no coordinados cuando camina o corre .....

\section{Subescala de Patrones Cognitivos}

La persona:

\begin{tabular}{ccc}
$\begin{array}{c}\text { Nunca } \\
\text { observado observado veces }\end{array}$ & $\begin{array}{c}\text { Algunas } \\
\text { veces } \\
\text { observado }\end{array}$ \\
& \multicolumn{3}{c}{ observ. }
\end{tabular}

Habla sobre un solo tema de manera excesiva.

$\begin{array}{llll}0 & 1 & 2 & 3\end{array}$

Demuestra habilidades o conocimientos superiores en temas o actividades específicas.

Emplea un lenguaje excesivamente preciso o pretencioso

\begin{tabular}{llll}
0 & 1 & 2 & 3 \\
0 & 1 & 2 & 3 \\
0 & 1 & 2 & 3 \\
0 & 1 & 2 & 3 \\
0 & 1 & 2 & 3 \\
0 & 1 & 2 & 3 \\
\hline
\end{tabular}

Otorga significados muy concretos a las palabras

Tiene dificultades para entender el humor o las bromas .

Demuestra una excelente memoria

Muestra un interés intenso y obsesivo en ciertos temas 


\section{Subescala de Habilidades Pragmáticas}

La persona:

\begin{tabular}{|c|c|c|c|c|}
\hline & $\begin{array}{c}\text { Nunca } \\
\text { observado }\end{array}$ & $\begin{array}{c}\text { Raras veces } \\
\text { observado }\end{array}$ & $\begin{array}{l}\text { Algunas } \\
\text { veces } \\
\text { observado }\end{array}$ & $\begin{array}{l}\text { Frec. } \\
\text { observ. }\end{array}$ \\
\hline 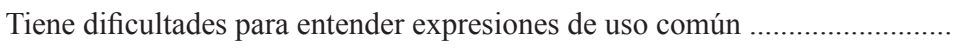 & 0 & 1 & 2 & 3 \\
\hline Tiene dificultades para identificar cuando alguien se está burlando ............... & 0 & 1 & 2 & 3 \\
\hline $\begin{array}{l}\text { Tiene dificultades para entender cuando él o ella está siendo ridiculizado, } \\
\text { expuesto o cuando se están divirtiendo a costa suya ........................................... }\end{array}$ & 0 & 1 & 2 & 3 \\
\hline $\begin{array}{l}\text { Tiene dificultades para entender el motivo por el cual él o ella no le agrada a } \\
\text { alguien }\end{array}$ & 0 & 1 & 2 & 3 \\
\hline No predice probables consecuencias en situaciones sociales .............................. & 0 & 1 & 2 & 3 \\
\hline 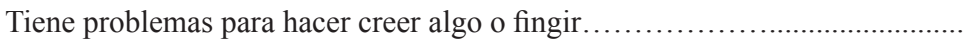 & 0 & 1 & 2 & 3 \\
\hline 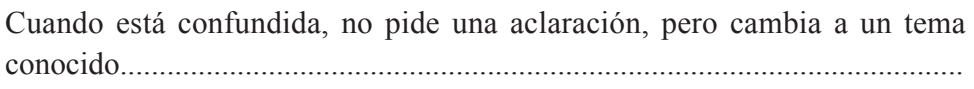 & 0 & 1 & 2 & 3 \\
\hline
\end{tabular}

